\title{
TU/e EN⿴HONE

\section{STM observations of GaAs(110) showing the top and bottom zig-zag rows of the surface}

\section{Citation for published version (APA):}

Raad, de, G. J., Bruls, D. M., Koenraad, P. M., \& Wolter, J. H. (2001). STM observations of GaAs(110) showing the top and bottom zig-zag rows of the surface. Physical Review B, 64(7), 075314-1/7. [075314].

https://doi.org/10.1103/PhysRevB.64.075314

DOI:

10.1103/PhysRevB.64.075314

Document status and date:

Published: 01/01/2001

\section{Document Version:}

Publisher's PDF, also known as Version of Record (includes final page, issue and volume numbers)

\section{Please check the document version of this publication:}

- A submitted manuscript is the version of the article upon submission and before peer-review. There can be important differences between the submitted version and the official published version of record. People interested in the research are advised to contact the author for the final version of the publication, or visit the $\mathrm{DOI}$ to the publisher's website.

- The final author version and the galley proof are versions of the publication after peer review.

- The final published version features the final layout of the paper including the volume, issue and page numbers.

Link to publication

\section{General rights}

Copyright and moral rights for the publications made accessible in the public portal are retained by the authors and/or other copyright owners and it is a condition of accessing publications that users recognise and abide by the legal requirements associated with these rights.

- Users may download and print one copy of any publication from the public portal for the purpose of private study or research.

- You may not further distribute the material or use it for any profit-making activity or commercial gain

- You may freely distribute the URL identifying the publication in the public portal.

If the publication is distributed under the terms of Article 25fa of the Dutch Copyright Act, indicated by the "Taverne" license above, please follow below link for the End User Agreement:

www.tue.nl/taverne

Take down policy

If you believe that this document breaches copyright please contact us at:

openaccess@tue.nl

providing details and we will investigate your claim. 


\title{
STM observations of GaAs(110) showing the top and bottom zig-zag rows of the surface
}

\author{
G. J. de Raad, D. M. Bruls, P. M. Koenraad, and J. H. Wolter \\ COBRA Inter-University Research Institute, Eindhoven University of Technology, P.O. Box 513, 5600 MB Eindhoven, The Netherlands
}

(Received 18 May 2000; revised manuscript received 19 March 2001; published 30 July 2001)

\begin{abstract}
We present an unusual image form of the GaAs(110) surface observed in cross-sectional scanning tunneling microscopy in UHV. It clearly shows the zig-zag row formed between the cations and anions in the top row of the GaAs(110) surface, as well as traces of the bottom row that lies between the top rows. It thereby closely resembles the true atomic lattice usually depicted by ball-and-stick models. We interpret the occurrence of this image form in terms of a chemical bond that forms between the apex of the tip and the nearest $\mathrm{Ga}$ atom in the top row of the sample surface. The formation of such a bond has recently been predicted theoretically. The results thus show that the tip-sample interaction, when it becomes significant, can give rise to entirely different and interesting image forms.
\end{abstract}

DOI: 10.1103/PhysRevB.64.075314

PACS number(s): 73.20.-r, 68.37.Ef, 73.61.Ey

\section{INTRODUCTION}

Since the work of Feenstra et al., ${ }^{1}$ the common way to interpret atomically resolved scanning tunneling microscopy (STM) images of GaAs(110) has been that the image shows either the $\mathrm{Ga}$ atoms or the As atoms in the top rows of the surface, depending on the polarity of the applied bias voltage (Fig. 1). At that time it was already known that the (110) surface of GaAs possesses four surface states, which are all located energetically outside the fundamental band gap of the semiconductor. ${ }^{2,3}$ Two of these states lie energetically inside the valence band $(A 5, A 4)$ and two lie inside the conduction band $(C 3, C 4)$. The same work also shows that the spatial distribution of the $A 5$ and $A 4$ states is centered on the toprow anions (As) of the surface, whereas the spatial distribution of the $C 3$ state is centered on the top-row cations $(\mathrm{Ga})$. The distribution of the $C 4$ state is centered mainly on the top-row cations, but the $C 4$ state also has some amplitude on the top-row anions as well as on both atom species in the bottom rows of the surface. ${ }^{2}$

The (110) surface of several III-V zinc-blende semiconductors was studied in more detail by Ebert et al., ${ }^{4}$ who showed that the different surface states each have a different spatial distribution within the surface unit cell. As a result, the atomically resolved STM image of such surfaces may change very significantly in appearance as a function of applied bias voltage. ${ }^{4,5}$ The (110) surface of InP has recently been described theoretically by Engels et $a .^{6}{ }^{6}$ in great detail. The work explains that the surface state usually designated as $C 4$ in reality consists of several empty surface resonances, each having a different spatial extent and slightly different energetic location. ${ }^{6}$ Close to the surface $\left(2.23 a_{0}=1.1 \AA\right.$ from the outwardly rotated $\mathrm{P}$ atoms), the $C 4$ state is centered on both the cations and anions and appears as a zig-zag row. Further away from the surface $\left(6.53 a_{0}\right.$ from the surface anion) the state shows up as maxima centered on the cations extending along $[\overline{1} 10] .{ }^{4,6}$ In the literature, several papers exist that report bias-voltage-dependent changes in the morphology of atomically resolved STM images of the GaAs(110) surface. These changes are interpreted in terms of the four surface states contributing in varying relative amounts to the total tunneling current. ${ }^{4,5,7}$ The usual TersoffHamann theory ${ }^{8,9}$ provides an adequate basis for this interpretation.

However, some types of atomic morphology are reported that have more than one atomic maximum per surface unit cell, and that therefore cannot be interpreted in terms of different surface states contributing to the total tunneling current. $^{10-12}$ In one such case, it is shown explicitly that the image form involves a reduced tip-sample distance. ${ }^{10}$ The result is interpreted in terms of tip-induced electronic states that are formed between the tip and sample as these two approach each other closely. ${ }^{10,13-16}$ At the same time, recent theoretical results indicate that a chemical bond may form between the apex atom of the tip and the nearest Ga atom in the top row of the sample surface, if the tip-sample distance is sufficiently reduced. ${ }^{17}$ It is thus clear that occasionally effects are observed in atomically resolved STM on $\mathrm{GaAs}(110)$ that cannot be explained within the framework of the usually applied Tersoff-Hamann theory.

In this paper, we report an unusual image form of the GaAs(110) surface that we have observed when imaging the empty states of the sample surface. It clearly shows the zigzag chain formed by the $\mathrm{Ga}$ atoms and As atoms in the top row, as well as traces of the second zig-zag chain formed by the $\mathrm{Ga}$ and $\mathrm{As}$ atoms in the bottom row of the surface. In doing so, it closely resembles the true atomic lattice which is

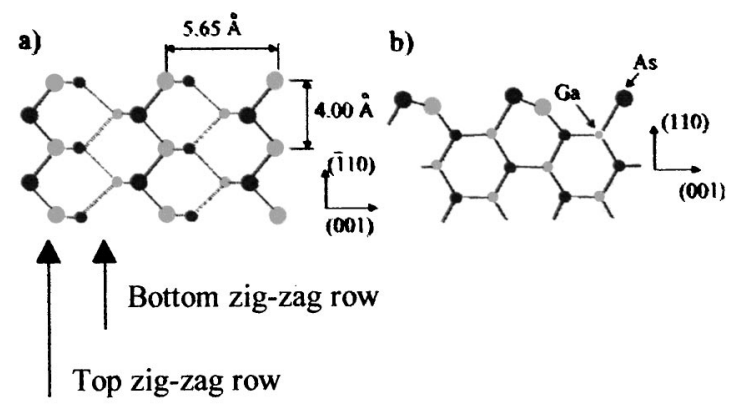

FIG. 1. (a) Relaxed GaAs(110) surface (top view). (b) Side view: The surface reconstructs by allowing the top-row As atoms to rotate outward and the top-row $\mathrm{Ga}$ atoms to move inward toward the bulk of the crystal. The process is known as "buckling." 
usually depicted by the ball-and-stick models of the GaAs(110) surface found in many textbooks. We think that this image form arises when a chemical bond forms between the tip and sample in the way that is described by Ke et al. ${ }^{17}$ To our knowledge, no STM images of GaAs(110) that show both the top and bottom rows of the surface have been reported in the literature until now.

\section{EXPERIMENT}

The STM work was performed in UHV (Omicron STM-1 setup operated at $4 \times 10^{-11}$ torr) on in situ cleaved GaAs samples. Doping concentration ${ }^{18}(\mathrm{Si})$ was $10^{18} \mathrm{~cm}^{-3}$. Our tips are etched electrochemically from $\varnothing .25 \mathrm{~mm}$ polycrystalline tungsten wire. After heating, $\mathrm{Ne}^{+}$ion self-sputtering, and bombardment by an $\mathrm{Ar}^{+}$ion gun, our tips routinely yield atomic resolution on $\mathrm{GaAs} / \mathrm{InP}(110)$ (apex radius $\sim 10 \mathrm{~nm}$ ). ${ }^{19}$ The bias voltage is applied to the sample. Our STM setup is equipped with a "dual mode" feature, which enables us to apply one set of scan parameters (bias voltage, tunneling current, feedback parameter) on the forward scan and another set of parameters on the backward one. We can thus image a given area of sample simultaneously for two different sets of parameters. All images shown in this paper are height images $[z(x, y)]$ that were obtained while operating the STM in constant-current mode.

We apply a background correction to the images that involves a "line-by-line tilt correction:" From the height along each fast scan line (horizontal in the images) a linear fit is made, which is then subtracted from the original height curve along that scan line. This same type of correction is commonly applied as an on-line correction during the acquisition of the data. In our measurements, the tip is usually scanned with a frequency between 2.5 and $5 \mathrm{~Hz}$. External vibrations with a frequency of a few hertz tend to raise or lower a fast scan line, and also to tilt it. When the scanning frequency of the tip is larger than four times the frequency of the external vibration, the vibration introduces only little curvature into the height profile along the scan line. This lineby-line correction is then very effective in filtering out the effects of such low-frequency external vibrations without otherwise altering the data. The effectiveness increases when the method is applied to smaller cutouts of a frame.

The same background correction can also be applied to all pixels lying in a line perpendicular to the fast scan direction (vertical in our images). Figures 2(b) and 2(c) below are cutouts from larger frames, where the top right corner is chosen inside a depression in the surface. The height surfaces $[z(x, y)]$ are thus warped instead of planar. We eliminate much of this warping by applying the line-by-line correction first in the horizontal direction and then in the vertical direction, which allows us to show details of the atomic lattice in all portions of the frame. For Figs. 3(c) and 3(d) this method is inapplicable since the dopants that are shown in the frame lie completely inside the frame. We have therefore used a band-pass fast Fourier transform filter to show details of the lattice.

Atomic resolution on semiconductor surfaces is usually understood in terms of electrons tunneling directly into or out of surface states. The STM images are then essentially microscopic maps that show the lateral variation in intensity of the density of states (DOS) associated with (combinations of) the surface states (Tersoff-Hamann theory). ${ }^{9}$ An important point in this theory is that the tunneling matrix element is proportional to the local sample surface DOS evaluated at the position of the center of the tip apex atom: The STM image shows the vacuum tails of the surface states. A direct consequence is that in the framework of the Tersoff-Hamann theory features in the sample DOS that lie within the surface layer do not appear in the STM image. We will refer to this process as "regular tunneling."

\section{TRUE LATTICE RESEMBLANT CORRUGATION}

The image form that we report occurs occasionally as an anomaly when we make voltage-dependent images of GaAs(110). So far, we have observed the image form, which we label "true lattice resemblant corrugation" [TLRC; see Fig. 2(a)], when imaging the empty states of $n$-GaAs(110) using a small value for the bias voltage $\left(0<V_{\text {bias }}<0.5 \mathrm{~V}\right)$. The image form is stable for a variable amount of time: It sometimes lasts no longer than several seconds or a minute, or it can last for up to $30 \mathrm{~min}$ as we have observed on one occasion. The TLRC image form can be reproduced, but we cannot obtain it at will or in a very controlled manner. The method that we apply to obtain the TLRC is by imaging the (110) surface of GaAs at positive sample voltage so as to produce normal-looking atomic resolution, which we can obtain routinely. From that point, we gradually decrease the applied bias voltage and/or increase the tunneling current, so as to decrease the tip-sample distance. Some of these attempts produce TLRC images; the others simply fail with a tip crash.

The appearance of TLRC always involves a sudden transition from the normal imaging mode, in which the atomically resolved lattice of the GaAs(110) surface appears as it usually does (see, e.g., Refs. 1, 5, and 19), to an anomalous mode that involves TLRC. An example of such a transition is shown in Fig. 2(c). In this particular case, the onset of TLRC involved a sudden transition from the normal morphology of the atomically resolved lattice (bottom of the frame) to an anomalous from that we have called the "preform" (top of the frame). TLRC itself occurred when the applied bias voltage was further reduced after the appearance of the preform. During other measurements, we have also observed transitions from the normal to the anomalous tunneling regime in which there was a direct change from the normallooking image to TLRC, without the preform appearing first. The change from the normal tunneling regime to the anomalous tunneling regime does not necessarily occur at the same moment that the bias voltage is changed: In Fig. 2(c), for instance, the transition occurred some time later, i.e., at a moment when the bias voltage was constant. The difficult part of obtaining TLRC is causing this transition to occur.

The image mode that we have called TLRC is shown in Fig. 2(a). The atomic lattice consists of parallel zig-zag rows ( $A$ and $B$ sites). Between these rows, a second set of zig-zag rows appears as well ( $C$ and $D$ sites). These rows lie $10 \mathrm{pm}$ 

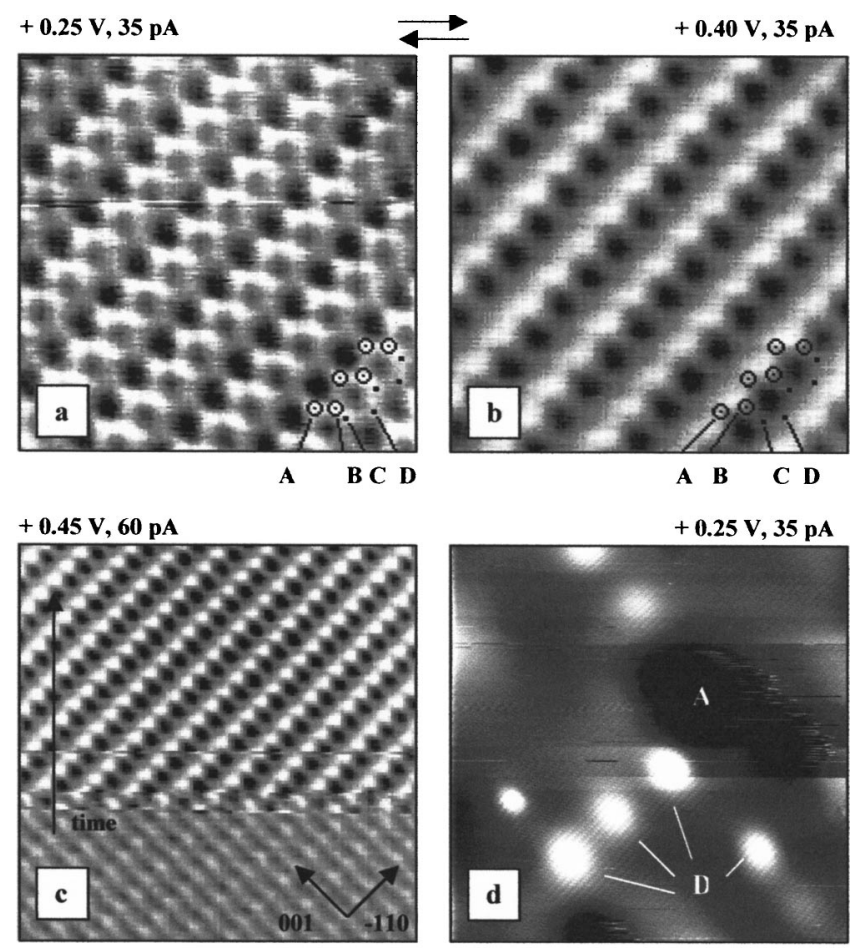

FIG. 2. (a) True lattice resemblant corrugation (TLRC) on $n$-GaAs $\left(3.2 \times 3.2 \mathrm{~nm}^{2}\right) . V=+0.25 \mathrm{~V}, I=35 \mathrm{pA}$. Gray scale $0-32$ $\mathrm{pm}$. The circles identify the top-row atoms $(A, B)$, the dots identify the bottom row atoms $(C, D)$. (b) Preform of TLRC. Frame size $3.2 \times 3.2 \mathrm{~nm}^{2}$, gray scale $0-41 \mathrm{pm}$. (a) and (b) have been obtained simultaneously using dual-mode imaging. The $A, B, C$, and $D$ sites of the TLRC have been projected into the image. (c) Transition from normal imaging to preform of TLRC (upper part). Slow scan direction is from bottom to top $(6.7 \times 6.7 \mathrm{~nm})$. Gray scale $0-39 \mathrm{pm}$. In the (raw) image, the atomic maxima of the preform lie $16 \mathrm{pm}$ higher than the atomic maxima in the bottom part of the image. It can be seen that the size of the surface unit cell associated with the preform is equal to the size of the unit cell of the normal atomic resolution shown in the bottom part of (c). (d) Large-scale image of the GaAs(110) surface showing TLRC. Frame size $35 \times 35 \mathrm{~nm}^{2}$. We interpret the bright features as Si dopants. The dark areas are interpreted as (negatively charged) adsorbates (Ref. 32).

below the rows belonging to the first set. This arrangement closely resembles the true atomic lattice shown in Fig. 1(a). It is possible to switch between TLRC [Fig. 2(a)] and its preform [Fig. 2(b)] by changing the bias voltage $(+0.25$ and $+0.45 \mathrm{~V}$ at $35 \mathrm{pA})$. Furthermore, the effect is very sensitive to the tip-sample distance: At $V=+0.35 \mathrm{~V}$, the image shows the preform with TLRC in the dark areas [Figs. 3(a) and 3(b)]. The change from preform to TLRC occurs over several nanometers $(36 \AA)$. When imaging a Si dopant, the dopant shows the preform while the surrounding lattice shows TLRC [Figs. 3(c), 3(d), and 2(d)]. It is thus clear that TLRC and the preform are related, and that the preform involves a slightly larger tip-sample distance than TLRC. As the fast scan direction is horizontal, the images also show very clearly that the transition between TLRC and its preform is reversible.

Close comparison of the lower and upper parts of Fig.

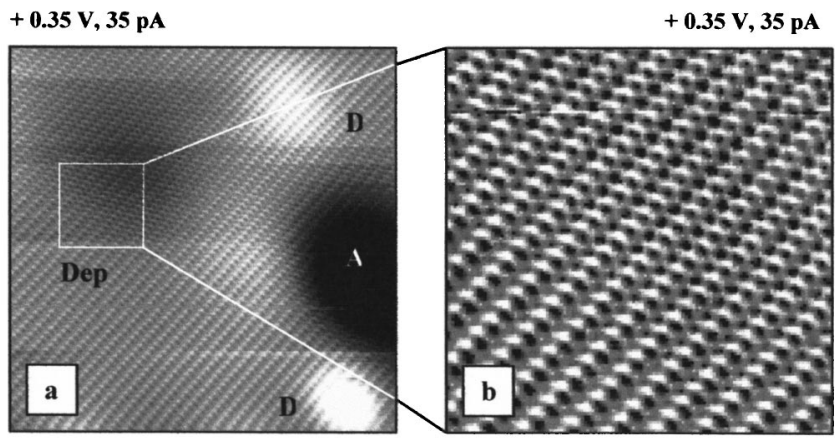

$+0.25 \mathrm{~V}, 35 \mathrm{pA}$

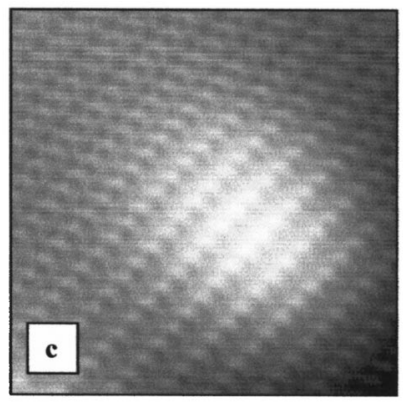

$+0.25 \mathrm{~V}, 35 \mathrm{pA}$ (FFT)

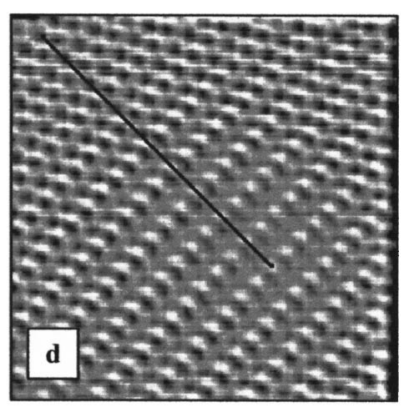

FIG. 3. (a,b) Transition from the preform to TLRC ( $V$ $=0.35 \mathrm{~V}, I=35 \mathrm{pA}$ ). Such transitions occur in areas where the local tunneling probability changes, which causes an adjustment in the tip-sample distance. The top right corner of (b) is part of a shallow, circular depression that lies 78-108 pm below the surrounding lattice (Dep). The depression is about $10 \mathrm{~nm}$ in diameter, and possibly represents a (negatively charged) subsurface $\mathrm{Si}_{\mathrm{As}}$ acceptor. Similar transitions from the preform to TLRC occur near adsorbates $(A)$. The bright features $(D)$ are interpreted as dopants. (a) $V$ $=+0.35 \mathrm{~V}, I=35 \mathrm{pA}$. Frame size $20 \times 20 \mathrm{~nm}^{2}$. (b) Frame size $6.7 \times 6.7 \mathrm{~nm}^{2}$, gray scale $0-34 \mathrm{pm}$. (c) High-resolution image showing one of the dopants shown in Fig. 2(d) in detail. Near the dopant the preform is seen while around the dopant the image shows TLRC. The apparent height of the dopant is $149 \mathrm{pm}$ with respect to the surrounding lattice. $V=+0.25 \mathrm{~V}, I=35 \mathrm{pA}$, frame size $6.25 \times 6.22 \mathrm{~nm}^{2}$, gray scale $0-199 \mathrm{pm}$. (d) Same as (c), after application of a bandpass fast Fourier transform filter. Gray scale 0-27 pm. A line drawn along [001] from the maxima of the preform coincides with the $B$ sites of the TLRC. From this we identify the $B$ sites as the Ga sites in the top row of the surface lattice.

2(c) shows that the $B$ sites in the preform coincide with the atomic maxima in the bottom part of Fig. 2(c). We can thus identify the $B$ sites in the preform [see Fig. 2(b)] as top-row Ga sites. A line drawn along [001] through the $B$ and $C$ sites in the preform would thus pass through the top-row Ga sites of the surface. From Fig. 2(b) it is evident that such a line would pass through the atomic maxima of the preform, as well as over the bridges connecting the $B$ and $C$ sites. We have drawn such a line from the central maximum of the dopant shown in Figs. 3(c) and 3(d). In Fig. 3(d) it can be seen that the line coincides with the $B$ sites of the TLRC, which we thus identify as $\mathrm{Ga}$ sites in the top row of the surface. The $A$ sites must then correspond to the As sites in the top row. 


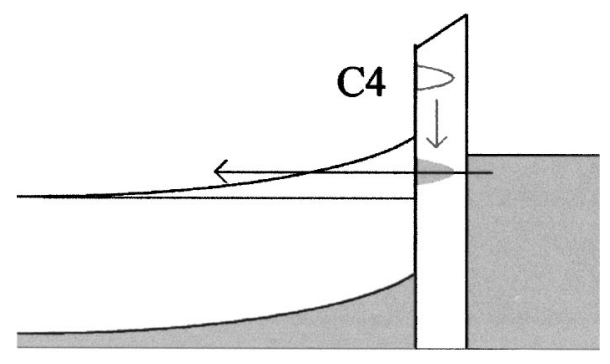

FIG. 4. Our interpretation of TLRC. Due to the tip-sample interaction, the buckling angle of the surface is decreased. This causes the $C 4$ state to come down in energy. Electrons can now tunnel resonantly through the displaced $C 4$ state, giving rise to TLRC.

\section{DISCUSSION}

This discussion consists of two parts. We will first discuss our own interpretation of the results presented in this paper. This interpretation assumes a shorter tip-sample distance than usual, resulting in the formation of a chemical bond between the tip and sample as described by Ke et al. ${ }^{17}$ The second part explores the plausibility of an alternative interpretation which assumes that the effect of TLRC is caused by an unusual density of states at the tip apex (tip DOS).

In earlier literature, STM images obtained in the filledstate mode showing both the Ga and As atoms in the top row of the surface were reported by Jäger et al. ${ }^{11}$ and by Heinrich et $a l .{ }^{10}$ In Ref. 10 it was shown that this type of imaging involves a significantly smaller tip-sample distance than usual. To our knowledge, however, no STM images also showing traces of the bottom zig-zag row have been reported in the literature. An image form similar to TLRC has also been observed on $\operatorname{InAs}(110) .^{12}$

From the model and experimental results to be presented in Ref. 5 concerning "normal'" atomically resolved images of $\operatorname{GaAs}(110)$, it is clear that for small positive sample bias the distance between the tunneling tip and an $n$-type sample becomes very sensitive to changes in the applied bias voltage. Also, the tip-sample distance can rapidly decrease to a few angstroms at small bias voltage. The voltage at which the rapid decrease in tip-sample distance sets in depends on the exact value of the tip work function. ${ }^{5}$ The tip work function, in its turn, varies from one experiment to the next and usually also changes when a spontaneous change in the tip DOS occurs. However, given the fact that TLRC occurs for low bias voltage, the hypothesis that the mechanism behind it involves a short tip-sample distance is logical.

As well as the likely tip-sample distances involved in the anomalous tunneling mode that produces TLRC and its preform, we also have to consider the effects of tip-induced band bending (TIBB). Feenstra and Stroscio ${ }^{20}$ have considered TIBB for a tunnel junction between a GaAs(110) surface and a $\mathrm{W}$ tip in detail. The tip work function is taken as $4.5 \mathrm{eV},{ }^{20}$ although the work function of a typical $\mathrm{W}$ tip can vary by about $0.3 \mathrm{eV} .^{21}$ According to Ref. 20, the TIBB is positive (i.e., the bands bend upward in energy-band diagrams such as Fig. 4) when a positive sample bias voltage is applied. Moreover, for small values of the applied bias voltage $\left(0<V_{\text {ample }}<0.75 \mathrm{~V}\right)$, the amount of TIBB is larger than the bias voltage itself. This leads to the situation that at small positive bias voltage the electrons tunnel from the tip through a surface depletion layer into the conduction-bandrelated bulk states ( $B C B$ states) of the sample. This surface depletion layer can be more than $10 \mathrm{~nm}$ thick. ${ }^{20}$ In a forthcoming publication ${ }^{5}$ we intend to present a study of the voltage-dependent changes in the morphology of the atomically resolved GaAs(110) surface in the "normal" tunneling regime. A necessary part of that work involves detailed consideration of the effects of TIBB occurring for a tunneling junction between a W tip and a GaAs(110) sample surface. Feenstra and Stroscio's notion that for $n$-type material and a tip work function of $4.5 \mathrm{eV}$ the electrons tunnel from the tip through a surface depletion layer into the bulk of the sample when a small, positive sample bias voltage is applied is fully consistent with our own results to be published in Ref. 5.

Recent theoretical calculations predict that the (110) surface of GaAs undergoes some very significant changes as it is being approached closely by a tip. The calculation was carried out in order to model the atomic resolution recently obtained in noncontact atomic force microscopy (AFM) on $\mathrm{GaAs}(110)$. Using a four-atom $\mathrm{Si}$ cluster to represent an AFM tip, it has been predicted by Ke et al. that the Ga relaxes outward under the influence of the tip-sample interaction. When the tip approaches the sample surface to within 3 $\AA$, a chemical bond may form between the Si apex atom and one of the $\mathrm{Ga}$ atoms in the top row of the surface. The vertical displacement of the $\mathrm{Ga}$ atom in this configuration can be up to $1 \AA$. This decreases or even partly reverses the buckling angle of the surface, since the displacement of the As atom for a tip-sample distance of $3 \AA$ is less than the displacement of the Ga atom. ${ }^{17}$ If the tip is withdrawn again, the tip-sample distance has to be increased to about $5 \AA$ before the bond between the apex atom and the Ga atom is ruptured. When, after the formation of the bond, the tip is approached closer still, the $\mathrm{Ga}$ atom and $\mathrm{Si}$ apex atom relax slightly further. For a tip-sample distance of less than about 2 $\AA$, the interaction becomes repulsive. ${ }^{17}$ The method used by Ke et al. was based on earlier work by Perez et al. ${ }^{22}$ in which the tip-sample interaction between a similar Si tip cluster and a $\mathrm{Si}(111)$ surface was modeled in great detail. A calculation similar to the one performed by Ke et al. has also been carried out for $\operatorname{InP}(110)$ by Tobik et al., ${ }^{23}$ showing that the relaxation of the $\operatorname{InP}(110)$ surface under the influence of tipsample interaction differs from the relaxation predicted for $\operatorname{GaAs}(110)$.

The decrease of the buckling angle by itself has a profound effect on the electronic structure of the GaAs(110) surface. As has been shown by Chelikowski and co-workers, the $C 3$ state moves up in energy whereas the $C 4$ state comes down. ${ }^{2,3}$ For the unrelaxed (truncated bulk) surface, the $C 4$ state lies energetically near the middle of the bulk band gap whereas the $C 3$ state lies in the bulk conduction band. The significance of this is that it thus becomes possible to image only the $C 4$ state at small positive bias voltage (and thus small tip-sample distance) due to the tip-induced structural modification of the sample surface. The $C 4$ state is localized on both the Ga and As atoms of the top zig-zag row, ${ }^{4,6}$ and also has a localization on the atoms of the bottom zig-zag 
row of the surface. ${ }^{2}$ References 4 and 6 predict that if the $C 4$ state is imaged at small tip-sample distance the top zig-zag row will appear in the images, showing both the top-row Ga and As atoms. If the localization of the $C 4$ state on the bottom zig-zag row were to show in the image as well, the result would be an image similar to TLRC. Due to the upward tip-induced band bending that we expect for $n$-GaAs being imaged at small positive sample bias, a significant semiconductor barrier (depletion layer) will be present between the tip and the bulk conduction-band states involved in the tunneling process. ${ }^{5} \mathrm{We}$ propose that the electrons tunnel resonantly through the $C 4$ surface state, so that the $C 4$ state spatially modulates the tunneling current so as to produce the TLRC. For this to occur, the $C 4$ state would have to lie energetically well inside the band gap, which is exactly the expected energetic location of the $C 4$ state if the buckling of the surface is eliminated due to the tip-sample interaction (see Fig. 4). It must be kept in mind, however, that the above considerations are based on calculations for the $\operatorname{GaAs}(110)$ surface with an altered surface reconstruction, while it is in all other respects assumed to be unperturbed. ${ }^{2-4,6}$ If the tipsample interaction has other significant effects besides a decrease of the buckling angle, the interpretation will become more complicated. A more rigorous treatment than given here will then be needed.

We have considered alternative possible explanations for TLRC. The most important one is the hypothesis that TLRC is caused by an unusual tip state. The central idea is that the tip DOS is such that the regular surface DOS of the GaAs(110) surface is imaged twice. If the two atomically resolved images are shifted with respect to each other, the resulting image might show two maxima and two minima per unit cell. An unfortunate arrangement of these two images could, in theory, result in an image similar to the one shown in Fig. 2(a). Although this mechanism would in our view fail to explain the observed dependence of TLRC on the tip-sample distance and bias voltage, the thought in itself is hardly surprising: The sudden transition shown in Fig. 2(c) looks very similar to the image obtained when a spontaneous tip change occurs during scanning.

For the images shown in Figs. 2 and 3, we can exclude the possibility that the tip used in the measurement had a double apex as the dopants $(D)$ and adsorbates $(A)$ visible in Fig. 2(d) do not appear twice in the image. This does not rule out the possibility that the tip apex ended in a microfacet consisting of several atoms rather than in one single atom. We suspect, however, that for most of our tips only one electronic state protruding from the tip apex is active in the tunneling process as we routinely obtain (normal) atomic resolution with our tips on GaAs(110) immediately after first tunneling contact ${ }^{19}$ [see also the bottom part of Fig. 2(c)].

For a tip that ends in a single atom, some electronic states exist that have two spatially separated lobes extending equally far toward the sample surface (see, e.g., the $d_{x z}$ state). ${ }^{9}$ Let us now consider the likelihood of occurrence and possible effects on the STM image of such a "double lobed" tip state with regard to TLRC. Based on the results of cluster $\left(\mathrm{W}_{4}\right.$ and $\left.\mathrm{W}_{5}\right)$ calculations ${ }^{9,24-26}$ it is generally agreed that for a tungsten tip ending in a single apex atom a $d_{z}^{2}$-like surface state dominates the tip DOS near the Fermi level. In the same energetic region a $d_{x y}$ state is also predicted, but this state carries only a fraction of the total tunneling current $\left(10^{-4}\right)$ when such a cluster is brought close to a model surface. Even when a tip-sample distance of $3 \AA$ is assumed in these calculations, the $d_{z}^{2}$ dominates the $d_{x y}$ state. According to Sachs and Noguera ${ }^{27} s$ - and $d$-type states dominate the DOS of a tungsten tip apex near the Fermi level, although a $p$-type state may dominate the tip DOS if a foreign atom is adsorbed on the apex.

A very important point has been made by Kobayashi and Tsukada $^{28}$ by simulating the tunneling process between a graphite surface and the bonding and antibonding molecular orbitals of the $\mathrm{H}_{2}$ molecule. If the active tunneling orbital has two spatially separated lobes that both carry a portion of the tunneling current, the resulting STM image need not be a superposition of the two independent images. The two tunneling currents interfere with each other. In the case of tunneling between the bonding orbital and the graphite surface, the calculated STM image is similar to the ideal STM image of graphite. ${ }^{28}$ For tunneling between the antibonding orbital and the graphite surface, the image becomes severely distorted, but does not lead to a doubling of the observed periodicity. ${ }^{28}$ A distorted image similar to the one found for the antibonding orbital is predicted for a $d_{z x}$-like orbital ${ }^{28}$ and for the tunneling active orbital of a $\langle 110\rangle$-oriented $\mathrm{W}$ tip with a missing apex atom. ${ }^{25,29}$

At first sight, these results appear to be in conflict with the experimental results reported by Giessibl et al. ${ }^{30}$ and by Park, Nogami, and Quate. ${ }^{31}$ The latter authors report the occurrence of two superimposed, independent images of the adatom pattern of the $(7 \times 7)$ reconstruction of $\mathrm{Si}(111)$. It must be noted, however, that the tips used in their experiment were used immediately after ac etching, without further in vacuo treatment. This means that their tips must certainly have had a thin oxide layer on them. An oxide layer with saturated bonds clearly does not represent the situation considered in Ref. 28. Furthermore, the adatoms in the $7 \times 7$ reconstruction of $\mathrm{Si}(111)$ lie relatively far apart $(7.68 \AA) .{ }^{31} \mathrm{It}$ is possible that the tip has imaged each of the adatoms separately, rather than having an interaction with the electronic structure of the surface lattice as is the case for tunneling on an atomically flat surface.

The results by Giessibl et al. ${ }^{30}$ concern frequencymodulation atomic force microscopy images of $\operatorname{Si}(111)-(7 \times 7)$. Giessibl et al. report the observation of a substructure in the images of the adatoms in the $\operatorname{Si}(111)-(7 \times 7)$ surface, which they attribute to the presence of a Si nanocluster on the apex of their tip, from which two spatially separated electronic lobes protrude. ${ }^{30}$ This result shows that the interference effect described by Kobayashi and Tsukada ${ }^{28}$ is a property of the tunneling current emanating from a double-lobed tip state: When, as is the case of AFM, the tip-sample interaction forces rather than the tunneling current is used as the basis of the feedback mechanism that controls the $z$ movement of the tip, the interference effect is apparently not observed.

We expect that our own tips, given the elaborate in vacuo 
treatment ${ }^{19}$ that we apply and the fact that we routinely obtain atomic resolution on $\operatorname{GaAs}\{110\},{ }^{5,19}$ are sharp and free of oxides. From the larger-scale images [Figs. 2(d) and 3(a)] it is clear that the tip used in our measurement did not have a double apex. According to calculations for a clean tungsten tip ending in a single apex atom, the presence of a tip state with two lobes extending to the surface does not lead to a superposition of two independent images. ${ }^{24-26,28}$ The same is true for a $\langle 110\rangle$-oriented tungsten tip that misses its apex atom and ends in a microfacet of four $\mathrm{W}$ atoms instead. ${ }^{25,29}$ We therefore cannot conceive how an unusual tip state could cause an apparent doubling of the lattice periodicity so as to produce an image similar to TLRC. This leaves the originally proposed explanation of TLRC in terms of a chemical bond forming between the tip apex atom and the $\mathrm{Ga}$ atoms in the top row of the surface as the remaining interpretation.

At this point, the physical meaning of atomically resolved STM images of the (110) surface of GaAs and other zincblende III-V semiconductors seems well understood as long as the tip-sample interaction remains small enough for the Tersoff-Hamann theory to apply. ${ }^{4,6}$ However, once the tipsample interaction does become significant, the images can change in drastic and poorly understood ways. Although the recent theoretical work on the interaction between a semiconductor surface and a nearby tip ${ }^{17,22,23}$ was meant to explain the recently obtained atomic resolution on zinc blende (110) surfaces by dynamic scanning force microscopy, ${ }^{33}$ it certainly has equal relevance to STM images as well. The occasional occurrence of TLRC and related image forms ${ }^{10-12}$ on such zinc blende (110) surfaces shows that the tip-sample interaction can profoundly influence the appearance of the atomically resolved lattice in STM. Although this influence is often unwanted, it can in principle also be used to obtain additional image forms. Understanding these image forms, and making use of the tip-sample interaction to obtain them, holds interesting challenges for theorists and experimentalists alike.

\section{SUMMARY AND CONCLUSION}

We report an unusual image form of the GaAs(110) surface that occasionally occurs when imaging the empty states of that surface. It closely resembles the true atomic lattice. We propose that the image form arises when a chemical bond forms between the tip and sample in the way that was predicted theoretically by Ke et al. ${ }^{17}$ This involves a significant reduction of the buckling angle of the surface. For the free GaAs(110) surface, such a reduction of the buckling angle is known to shift the $C 3$ and $C 4$ surface states, causing the $C 4$ state to lie well inside the band gap of the semiconductor. ${ }^{2}$ If electrons then tunnel resonantly through the shifted $C 4$ state, the resulting image will show the localization of the $C 4$ state within the surface layer, resulting in TLRC.

We have considered the possibility that the image form we observed might have been caused by an unusual electronic state present at the apex of the tunneling tip. From simulations of tunneling between a multilobed tip state and a sample surface that are reported in the literature, it seems unlikely that such a tip state will lead to an image form with more than one atomic maximum per surface unit cell. ${ }^{28}$ The results give a clear perspective on a very interesting way to image the (110) surface of GaAs.

\section{ACKNOWLEDGMENTS}

We gratefully acknowledge Dr. Martin Wenderoth, Dr. Ruben Perez, and Dr. An DePuydt for stimulating discussions. This work is part of the research program of the "Stichting Fundamenteel Onderzoek der Materie" (FOM), which is financially supported by the "Nederlandse organisatie voor Wetenschappelijk Onderzoek" (NWO).
${ }^{1}$ R. M. Feenstra, J. A. Stroscio, J. A. Tersoff, and A. P. Fein, Phys. Rev. Lett. 58, 1192 (1985).

${ }^{2}$ J. R. Chelikowski and M. L. Cohen, Phys. Rev. B 20, 4150 (1979).

${ }^{3}$ J. R. Chelikowski, S. G. Louie, and M. L. Cohen, Phys. Rev. B 14, 4724 (1976).

${ }^{4} \mathrm{Ph}$. Ebert, B. Engels, P. Richard, K. Schroeder, S. Blügel, C. Domke, M. Heinrich, and K. Urban, Phys. Rev. Lett. 77, 2997 (1996).

${ }^{5}$ G. J. de Raad, D. M. Bruls, P. M. Koenraad, and J. H. Wolter (unpublished).

${ }^{6}$ B. Engels, P. Richard, K. Schroeder, S. Blügel, Ph. Ebert, and K. Urban, Phys. Rev. B 58, 7799 (1998).

${ }^{7}$ C. S. Jiang, T. Nakayama, and M. Aono, Jpn. J. Appl. Phys., Part 2 36, L1336 (1997).

${ }^{8}$ J. Tersoff and D. R. Hamann, Phys. Rev. Lett. 50, 1998 (1983); Phys. Rev. B 31, 805 (1985).

${ }^{9}$ C. J. Chen, Introduction to Scanning Tunneling Microscopy (Oxford University Press, Oxford, 1993). Chapter 3 explains that for an $s$-like tip state the tunneling matrix element is proportional to the surface DOS of the sample evaluated at the center of the tip apex atom. The usual interpretation of STM results is well summarized by Eqs. (2.36) and (3.26).

${ }^{10}$ A. J. Heinrich, M. Wenderoth, M. A. Rosentreter, M. A. Schneider, R. G. Ulrich, Appl. Phys. Lett. 70, 449 (1996). See also $\mathrm{Ph}$. D. thesis, A. J. Heinrich, Cuvillier Verlag, Göttingen, Germany, 1998. The thesis shows that for small tip-sample distance the band gap evident in $I(V)$ spectra taken at normal tipsample distances disappears.

${ }^{11}$ N. D. Jäger, X. Liu, J. F. Zheng, N. Newman, D. F. Olgletree, E. R. Weber, and M. Salmeron, in Proceeding of the 23rd International Conference on the Physics of Semiconductors, edited by M. Scheffler and R. Zimmerman (World Scientific, Berlin, 1996), pp. 847-850.

${ }^{12}$ A. Depuydt et al. (unpublished).

${ }^{13} \mathrm{~S}$. Ciraci, in Scanning Tunneling Microscopy III, edited by R. Wiesendanger and H.-J. Günteroth (Springer-Verlag, Berlin, 1996), pp. 182-197. 
${ }^{14}$ S. Ciraci, A. Baratoff, and I. P. Batra, Phys. Rev. B 42, 7618 (1990).

${ }^{15}$ S. Ciraci, A. Baratoff, and I. P. Batra, Phys. Rev. B 41, 2763 (1990).

${ }^{16}$ E. Tekman and S. Ciraci, Phys. Rev. B 40, 10286 (1989).

${ }^{17}$ S. H. Ke, T. Uda, R. Perez, I. Stich, and K. Terakura, Phys. Rev. B 60, 11639 (1999). In the work presented in Refs. 17, 22 and 23 , the tip is represented by a four-atom Si tetrahedron. On the back side of the tetrahedron, the dangling bonds have been saturated with hydrogen atoms.

${ }^{18}$ We use commercially available $n$-doped GaAs wafers with a nominal doping concentration of $1 \times 10^{18} \mathrm{~cm}^{-3}$. The actual doping concentration may lie between 1 and $2 \times 10^{18} \mathrm{~cm}^{-3}$.

${ }^{19}$ G. J. de Raad, P. M. Koenraad, and J. H. Wolter, J. Vac. Sci. Technol. B 17, 1946 (1999).

${ }^{20}$ R. M. Feenstra and J. A. Stroscio, J. Vac. Sci. Technol. B 5, 923 (1987).

${ }^{21}$ R. Dombrowdki, C. Steinebach, C. Wittneven, M. Morgenstern, and R. Wiesendanger, Phys. Rev. B 59, 8043 (1999).

${ }^{22}$ R. Perez, I. Stich, M. C. Payne, and K. Terakura, Phys. Rev. B 58, 10835 (1998).
${ }^{23}$ J. Tobik, I. Stich, R. Perez, and K. Terakura, Phys. Rev. B 60, 11639 (1999).

${ }^{24}$ S. Ohnishi and M. Tsukada, Solid State Commun. 71, 391 (1989).

${ }^{25}$ M. Tsukada, K. Kobayashi, and S. Ohnishi, J. Vac. Sci. Technol. A 8, 160 (1990).

${ }^{26}$ M. Tsukada, N. Shima, S. Ohnishi, and Y. Chiba, J. Phys. (Paris), Colloq. 48, C6-91 (1987).

${ }^{27}$ W. Sachs and C. Noguera, Phys. Rev. B 43, 11612 (1991).

${ }^{28}$ K. Kobayashi and M. Tsukada, J. Phys. Soc. Jpn. 58, 2238 (1989).

${ }^{29}$ M. Tsukada, K. Kobayashi, and N. Isshiki, Surf. Sci. Rep. 13, 265 (1991).

${ }^{30}$ F. J. Giessibl, S. Hembacher, H. Bielefeldt, and J. Mannhart, Science 289, 422 (2000).

${ }^{31}$ S. I. Park, J. Nogami, and C. F. Quate, Phys. Rev. B 36, 2863 (1987).

${ }^{32}$ J. A. Stroscio, R. M. Feenstra, and A. P. Fein, Phys. Rev. Lett. 58, 1668 (1987).

${ }^{33}$ A. Schwarz, W. Allers, U. D. Schwarz, and R. Wiesendanger, Appl. Surf. Sci. 140, 293 (1999). 